\title{
L'utilisation de l'hydrolyse enzymatique pour la production de nanocellulose dans une stratégie de bioraffinage forestier intégré (synthèse bibliographique)
}

\author{
Pierre-Louis Bombeck ${ }^{(1)}$, Jacques Hébert ${ }^{(1)}$, Aurore Richel ${ }^{(2)}$ \\ (1) Université de Liège - Gembloux Agro-Bio Tech. Département BIOSE. Unité de Gestion des Ressources forestières et des \\ Milieux naturels. Passage des Déportés, 2. BE-5030 Gembloux (Belgique).E-mail : pl.bombeck@ulg.ac.be \\ (2) Université de Liège - Gembloux Agro-Bio Tech. Département AgroBioChem. Unité de Chimie biologique et industrielle. \\ Passage des Déportés, 2. BE-5030 Gembloux (Belgique).
}

Reçu le 30 mars 2015, accepté le 27 novembre 2015.

Introduction. Dans un monde qui cherche à se défaire de sa dépendance à la pétrochimie, le concept de bioraffinage de la biomasse forestière est de plus en plus étudié. Dans une recherche de valorisation maximale des composants de cette biomasse, la transformation de fibres de cellulose en nanocellulose séduit de plus en plus l'industrie papetière par sa haute valeur ajoutée. Littérature. Le concept de bioraffinage forestier intégré vise l'adaptation des usines de pâte à papier en bioraffineries où un maximum de coproduits sont valorisés. Au départ de la cellulose contenue dans la pâte, deux types de nanocellulose peuvent être obtenus en déstructurant les fibres selon différents moyens. Comme elle génère des coproduits valorisables, l'hydrolyse enzymatique constitue un moyen de production de nanocellulose mais doit néanmoins être combinée à des traitements mécaniques. La production de nanocellulose au départ de pâte à papier chimique est une étape de fin de chaine qui peut même s'envisager sans modifier le fonctionnement des usines actuelles. La viabilité économique de cette production et la taille du marché potentiel sont l'objet d'études récentes qui s'avèrent encourageantes.

Conclusions. La nanocellulose est un biomatériau d'avenir dont le domaine d'application ne cesse de grandir. La cellulose contenue dans la pâte à papier peut servir de matière première à la production de nanocellulose à l'aide de prétraitements enzymatiques générateurs de coproduits valorisables. Cette production peut s'intégrer dans une usine chimique classique de pâte et constituer ainsi un élargissement de la gamme de produits proposés par l'industrie papetière.

Mots-clés. Pâte cellulosique, nanocellulose, produit cellulosique, hydrolyse, hydrolyse enzymatique, biomasse, industrie de pâte et papier, bioraffinage forestier.

\section{Enzymatic hydrolysis to produce nanocellulose in an integrated forest biorefinery strategy. A review}

Introduction. In a world that seeks to break free from petrochemicals, the concept of forest biomass biorefinery is increasingly being studied as a way to maximize the value of the components of this biomass. Due to the high added value expected when transforming cellulose fibers into nanocellulose, this technology is highly attractive to the pulp and paper industrial world. Literature. The concept of integrated forest biorefinery is to adapt existing pulp mills so as to maximize the value of the co-products. Through the use of various methods, two types of nanocellulose may be obtained from the cellulose in the pulp. Due to its production of valuable byproducts, enzymatic hydrolysis is an interesting method to use for this purpose, but it must be combined with mechanical post-treatments. The production of nanocellulose from chemical pulp takes place in the final step of pulp production, and could be implemented without modifying the existing mills. The economic viability of this mode of production and the potential market size are the subject of recent studies that appear encouraging.

Conclusions. Nanocellulose is a promising biomaterial whose field of application continues to grow. Cellulose in wood pulp can be used as a raw material for the production of nanocellulose using enzymatic pre-treatments that generate valuable co-products. This process can be integrated into a conventional chemical pulp mill and constitutes a broadening of the products available to the pulp and paper industry.

Keywords. Pulp, nanocellulose, cellulose products, hydrolysis, enzymatic hydrolysis, biomass, pulp and paper industry, forest biorefinery. 


\section{INTRODUCTION}

La raréfaction attendue des ressources fossiles et la volonté internationale de réduire les émissions de $\mathrm{CO}_{2}$ amènent de plus en plus la recherche à s'orienter vers des solutions alternatives à la production d'énergie et aux produits issus classiquement de la pétrochimie. Tout en prenant en compte ces considérations, l'industrie papetière, secteur exigeant des investissements initiaux lourds, est également à la recherche de nouveaux produits et procédés permettant l'exploitation et la valorisation la plus judicieuse de l'intégralité des constituants du bois qu'elle consomme. C'est dans ce contexte qu'émerge le concept de bioraffinerie forestière intégrée (Zhang et al., 2011 ; Browne et al., 2013 ; Christopher, 2013). Ce type de bioraffinerie, alimentée par la biomasse lignocellulosique forestière et dont l'infrastructure s'appuie sur les installations d'une usine de pâte chimique, cherche à valoriser au maximum tous les composants de cette biomasse à travers des procédés à faible impact environnemental. Le principal constituant de cette biomasse forestière est la cellulose, dont les principaux usages en termes de volumes restent dans le domaine des pâtes et papiers (FAO, 2013). Il est cependant possible de déconstruire la structure des fibres de cellulose afin d'en libérer les éléments dont le diamètre va jusqu'à l'échelle nanométrique (Brinchi et al., 2013 ; Dufresne, 2013 ; Isogai, 2013). On obtient alors une gamme de produits, aux propriétés différentes des fibres proprement dites, dont l'appellation varie avec les dimensions de ceux-ci. On distingue déjà ainsi, selon le degré de déconstruction, les filaments de cellulose (FiloCell ${ }^{\mathrm{TM}}$ ), d'un diamètre allant de 30 à $500 \mathrm{~nm}$ et d'une longueur de 100 à $2000 \mu \mathrm{m}$, et les nanocelluloses à proprement parler (Tableau 1). Les propriétés particulières de ces nanocelluloses permettent une variété d'usages dans des domaines à haute valeur ajoutée, allant du secteur pharmaceutique à l'aérospatial (Dufresne, 2013 ; Cowie et al., 2014 ; Shatkin et al., 2014). Cette valorisation supérieure de la cellulose peut s'associer avec les objectifs visés par le bioraffinage de la biomasse forestière. En effet, les procédés de production de nanocellulose utilisent habituellement une cellulose d'une pureté élevée (Brinchi et al., 2013). Cela s'explique par l'influence des hémicelluloses et lignines résiduelles, qui forment alors une barrière limitant l'accès des réactifs à la cellulose et augmentent également la consommation de réactifs et d'énergie pendant le processus de séparation (Jonoobi et al., 2015). Notons que la présence d'hémicelluloses peut être aussi vue comme un avantage car favorisant alors par la suite l'individualisation des nanofibres produites (Chaker et al., 2013). La séparation des différents constituants de la biomasse lignocellulosique, suivie de leur valorisation optimale telle qu'envisagée dans une bioraffinerie forestière intégrée, en fait une source de cellulose idéale pour cette filière. Dans certains pays, les techniques de production actuelles de nanocellulose font parfois appel à des procédés chimiques qui peuvent s'avérer lourds et peu écologiques sans contrôles stricts et si aucune optique de recyclage n'est envisagée. D'autres pays, comme le Canada, s'orientent ainsi vers des procédés avec des empreintes environnementales globales plus faibles en employant, par exemple, des techniques d'hydrolyse en conditions acides très contrôlées ou bien mécano-enzymatiques. La présente étude fait le point sur le potentiel d'utilisation de l'hydrolyse par voie enzymatique en tant qu'un de ces traitements, apportant ainsi une plus-value à la cellulose et pouvant s'intégrer également dans le cadre d'une bioraffinerie forestière intégrée.

\section{LA CELLULOSE}

La cellulose est le biopolymère naturel et renouvelable le plus abondant sur terre. Elle provient majoritairement de la paroi cellulaire des plantes (le bois, source principale de cellulose, en contient de 40 à $50 \%$, le coton $90 \%$, le lin $80 \%$ et le chanvre $70 \%$ [Stevanovic et al., 2009]), mais peut aussi être d'origine bactérienne (Acetobacter xylinum) ou animale (chez les invertébrés marins du sous-embranchement des

Tableau 1. Les différents types de nanocelluloses (d'après Klemm et al., 2011 ; Dufresne, 2012) - The different types of nanocelluloses (adapted from Klemm et al., 2011; Dufresne, 2012).

\begin{tabular}{lll}
\hline Type de nanocellulose & Synonymes & Taille moyenne \\
\hline Microfibrillatted cellulose (MFC) & $\begin{array}{l}\text { Nanofibrils, microfibrils, cellulose nanofibers } \\
\text { (CNF), nanofibrillated cellulose (NFC) }\end{array}$ & $\begin{array}{l}\text { Diamètre : 5-100 nm } \\
\text { Longueur : plusieurs micromètres }\end{array}$ \\
Cellulose nanocrystals (CNC) & $\begin{array}{l}\text { Nanocrystalline cellulose (NCC), crystallites, } \\
\text { whiskers (Wh), cellulose nanowhiskers (CNW), } \\
\text { rodlike cellulose microcrystals }\end{array}$ & $\begin{array}{l}\text { Diamètre : 5-70 nm } \\
\text { Longueur : 100-250 nm }\end{array}$ \\
Bacterial nanocellulose (BNC) & $\begin{array}{l}\text { Bacterial cellulose, microbial cellulose, } \\
\text { biocellulose }\end{array}$ & $\begin{array}{l}\text { Diamètre : 20-100 nm } \\
\text { Différents types de réseaux de } \\
\text { nanofibres }\end{array}$ \\
\hline
\end{tabular}


Tuniciers ou Urochordés) (Klemm et al., 2011). On estime la production mondiale annuelle de cellulose par les plantes à plus de $50 \cdot 10^{9} \mathrm{t}$ et la ressource totale dépasserait les $10^{11} \mathrm{t}$ (Habibi et al., 2010). Les forêts regroupant $80 \%$ de cette cellulose, le bois représente la source principale de cellulose sur terre (Stevanovic et al., 2009).

La cellulose est le constituant principal du bois (Tableau 2) où elle forme l'élément structurant des parois cellulaires et joue le rôle de renfort axial. Elle se présente sous la forme de fibres de longueurs variables. De manière simplifiée, ces fibres sont ellesmêmes composées de microfibrilles de cellulose, dont l'orientation et le degré de cristallinité déterminent les propriétés chimiques, physiques et mécaniques

Tableau 2. Constituants majeurs du bois - Major constituents of wood.

\begin{tabular}{ll}
\hline Composants du bois & Contenu $(\%)$ \\
\hline Cellulose & $40-50$ \\
Hémicelluloses & $15-35$ \\
Lignines & $20-30$ \\
Extractibles & $1-5$ \\
\hline
\end{tabular}

du bois. Les microfibrilles sont caractérisées par un diamètre variant de 10 à $25 \mathrm{~nm}$ (selon l'origine de la cellulose) et une longueur pouvant atteindre plusieurs dizaines de micromètres. Ces microfibrilles sont composées de fibrilles dites «élémentaires ", d'un diamètre moyen de $3,5 \mathrm{~nm}$, formées de chaines de cellulose (homopolysaccharide linéaire formé par la condensation de D-glucopyranose), unies par des liaisons $\beta(1 \rightarrow 4)$ [Brinchi et al., 2013; Fengel et al., 1989]) (Figure 1). Ces chaines de cellulose ne sont pas individuellement isolées, mais assemblées et liées (tant au niveau intra- que intermoléculaire) par des liaisons hydrogènes. Ces liaisons résultent de l'interaction physico-chimique entre les groupements hydroxyles de chaines macromoléculaires voisines (Stevanovic et al., 2009). Une hiérarchie a été identifiée et définie depuis longtemps, dans laquelle les fibrilles élémentaires s'assemblent en plus larges unités appelées microfibrilles, elles-mêmes assemblées entre elles et formant les fibres de cellulose (Fengel et al., 1989). Il s'avère que le caractère ordonné des molécules n'est pas uniforme dans les microfibrilles, formant ainsi des zones cristallines (zones ordonnées) et des zones amorphes (zones peu ordonnées) (Moon et al., 2011). Dans le bois comme chez les plantes, la cellulose est présente en association avec d'autres biopolymères
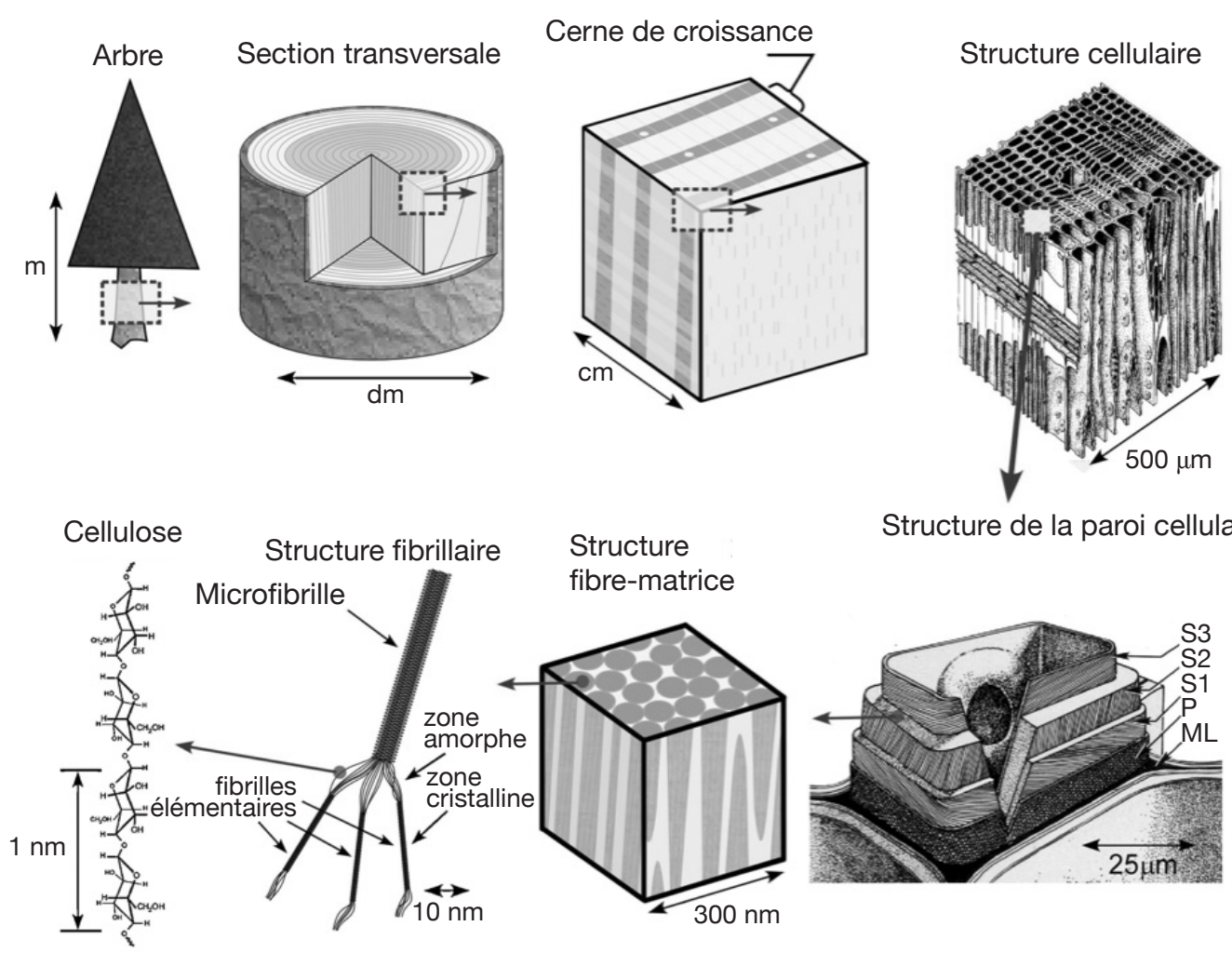

Structure de la paroi cellulaire

Figure 1. Structure hiérarchique du bois : de l'arbre à la cellulose (d'après Postek et al., 2011. C) IOP Publishing. Reproduit avec la permission d'IOP Publishing. Tous droits réservés) - Hierarchical structure of wood: from tree to cellulose (adapted from Postek et al., 2011. (C) IOP Publishing. Reproduced by permission of IOP Publishing. All rights reserved). 
tels que les hémicelluloses et les lignines, formant des complexes lignine-polysacharides (en anglais lignincarbohydrates complex [LCC]) (Fengel et al., 1989; Stevanovic et al., 2009 ; Christopher, 2013).

\section{BIORAFFINERIE FORESTIÈRE INTÉGRÉE À UNE USINE DE PÂTE CHIMIQUE TYPE «KRAFT »}

Avec le souci actuel de rechercher des alternatives ou compléments aux ressources fossiles, le concept de bioraffinerie forestière intégrée est de plus en plus mis en avant (Christopher, 2013 ; Rafione et al., 2014). Comme pour la bioraffinerie, il s'agit d'installations qui intègrent les équipements et les procédés durables destinés à convertir la biomasse (ici forestière) en un large spectre de bioproduits à haute valeur ajoutée d'une part et, d'autre part, en bioénergie (bioéthanol, chaleur, électricité). Le concept est analogue aux raffineries pétrolières qui génèrent une variété de produits et de sources d'énergie à partir du pétrole. Il s'agit de tirer avantage au maximum de la variété de composants et d'intermédiaires de la biomasse pour en maximiser la valeur ajoutée. Cette approche multidisciplinaire intègre des procédés de conversion physiques, mécaniques, chimiques, biologiques, des catalyses et biocatalyses pour augmenter l'efficience, diminuer les couts et la consommation d'énergie. Dans le cas d'une bioraffinerie forestière intégrée (Integrated forest biorefinery, IFBR), c'est la biomasse lignocellulosique forestière (bois et résidus forestiers) qui est utilisée. Comme cette biomasse lignocellulosique est constituée majoritairement de quatre composants (Tableau 2), une IFBR intègre donc quatre plateformes dans une optique de production de bioproduits et biocarburants issus de ces quatre composants (Figure 2).

Les usines de pâte chimique fonctionnant déjà comme des bioraffineries forestières élémentaires, elles constituent une base de départ idéale pour leur évolution complète en IFBR (Zhang et al., 2011 ; Bajpai, 2013; Machani et al., 2014). En effet, l'industrie papetière (pâte et papier) est une des plus importantes industries de biomasse non alimentaire au niveau mondial, avec une capacité de production de pâte d'environ 180 millions de tonnes par an (FAO, 2013). Cependant, l'évolution négative du marché papetier au niveau mondial, à l'exception des secteurs de l'emballage et des papiers domestiques et sanitaires, oblige ces industries à réduire encore leurs couts de fabrication, mais surtout à identifier de nouveaux débouchés et à générer de nouveaux revenus issus de ces nouveaux produits. Parmi les usines de production de pâte à papier, celles employant les procédés dits « chimiques » sont parfaitement indiquées pour jouer la transition de l'usine de pâte « classique » à l'IFBR. Ces usines, via leurs procédés, produisent et valorisent déjà une partie des coproduits issus de la fabrication de la pâte. Le procédé chimique le plus employé dans le monde est le procédé au sulfate, produisant une pâte dite «Kraft». Les installations employant ce procédé sont particulièrement bien adaptées pour évoluer de plus en plus vers une IFBR (Moshkelani et al., 2013; Rafione et al., 2014). Le procédé Kraft consiste en la cuisson, à haute température (170$175^{\circ} \mathrm{C}$ ) et pendant une durée de 2 à $5 \mathrm{~h}$, des copeaux

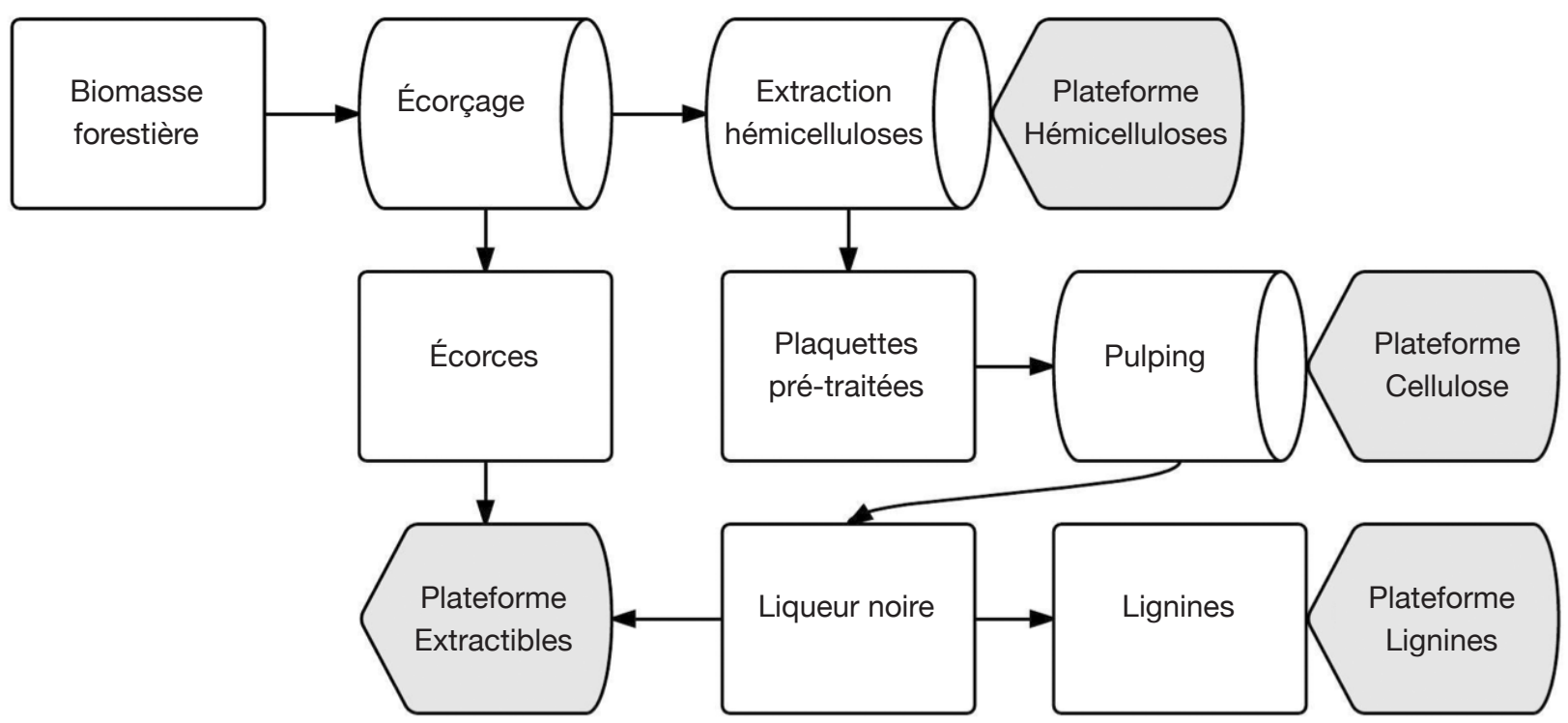

Figure 2. Schéma synthétique des processus et plateformes impliqués dans une Bioraffinerie Forestière Intégrée (IFBR) (d'après Christopher, 2013) - Synthetic scheme of processes and platforms involved in an Integrated Forest Biorefinery (IFBR) (adapted from Christopher, 2013). 
de bois dans un mélange d'hydroxyde de sodium et de sulfure de sodium. Cette cuisson provoque la dissolution de la majeure partie des lignines et hémicelluloses contenues dans le bois pour récupérer in fine des fibres de celluloses relativement intactes. Ces fibres contiennent néanmoins encore des teneurs non nulles de lignines et d'hémicelluloses. Outre cette dissolution, le procédé Kraft se distingue par le taux élevé de récupération $(98 \%)$ des réactifs chimiques utilisés. Cette récupération passant par une étape de combustion des lignines et hémicelluloses dissoutes, les usines de pâtes Kraft sont actuellement capables, par cogénération, d'assurer leur autosuffisance énergétique et d'en dégager un surplus. Un procédé de production de fibres déjà fortement optimisé et qui permet une valorisation des coproduits, des installations existantes et performantes, une main-d'œuvre spécialisée, une logistique d'approvisionnement en matières premières et de traitement des effluents déjà en place font des usines de pâtes chimiques le point de départ idéal à l'évolution de ces installations en IFBR. Bien que les usines de production de pâtes chimiques selon le procédé sulfite puissent être également des exemples de bioraffineries intégrées, l'utilisation prédominante du procédé Kraft dans le monde (près de $90 \%$ des pâtes chimiques [Christopher, 2013 ; FAO, 2013]), ainsi que la qualité différente des lignines qui peuvent en être récupérées, semble en faire le premier choix en termes de conversion totale vers une IFBR (Bajpai, 2013 ; Moshkelani et al., 2013 ; Rafione et al., 2014).

\section{LA NANOCELLULOSE ISSUE DE PÂTE À PAPIER}

$\mathrm{Si}$, actuellement, la principale application en termes de volume (FAO, 2013) de la pâte de cellulose est son utilisation pour la fabrication des papiers et cartons, une autre application porteuse à plus haute valeur ajoutée est étudiée depuis plusieurs années : utiliser une partie de la pâte produite en tant que matière première pour la fabrication de nanocellulose (Henriksson et al., 2007 ; Pääko et al., 2007 ; Spence et al., 2010 ; Mishra et al., 2012). En effet, en arrivant à en extraire de la cellulose à une échelle nanoscopique, la majorité des imperfections associées à la structure hiérarchique des fibres disparait (Moon et al., 2011). Cette nanocellulose possède des caractéristiques mécaniques, chimiques et optiques largement étudiées qui ouvrent la voie à de nombreux usages allant des applications directes dans l'industrie papetière aux biocomposites, en passant par les domaines tels que le pharmaceutique et le biomédical (Habibi et al., 2010 ; Siró et al., 2010 ; Klemm et al., 2011 ; Moon et al., 2011 ; Brinchi et al., 2013 ; Dufresne, 2013 ; Isogai, 2013 ; Lindström et al., 2014 ; Jorfi et al., 2015).
Le terme «nanocellulose» fait généralement référence à un matériau cellulosique dont au moins une des dimensions est inférieure à $100 \mathrm{~nm}$. Selon les dimensions et le procédé de fabrication, on distingue la nanocellulose selon plusieurs types (Klemm et al., 2011 ; Dufresne, 2012). La cellulose microfibrillée (microfibrillated cellulose, MFC) provient de la délamination de pâte de bois par pression mécanique en combinaison avec un traitement chimique ou enzymatique. La cellulose nanocristalline (cellulose nanocrystals, CNC) est obtenue par hydrolyse de cellulose pouvant provenir de plusieurs sources. Notons également la nanocellulose bactérienne (bacterial nanocellulose, BNC) synthétisée par certaines bactéries du genre Gluconacetobacter. Selon la littérature, ces différents types de nanocellulose peuvent être nommés différemment. Des recueils non exhaustifs des termes anglophones utilisés ont été proposés par Klemm et al. (2011) et Dufresne (2012) (Tableau 1).

\subsection{Cellulose micro-fibrillée (MFC)}

La cellulose micro-fibrillée (microfibrillated cellulose, MFC) est obtenue par destruction mécanique de l'organisation naturelle des fibres de cellulose en soumettant une suspension de pâte à des forces de cisaillement très élevées. Une fois la structure originale de la fibre déconstruite, on obtient alors des microfibrilles (seules ou en paquets) d'un diamètre de l'ordre de 10 à $100 \mathrm{~nm}$ et d'une longueur pouvant atteindre plusieurs micromètres (Klemm et al., 2011 ; Dufresne, 2012). Différents traitements mécaniques peuvent être utilisés pour cette déconstruction. Taniguchi et al. (1998) et Iwamoto et al. (2007) ont ainsi obtenu de la MFC par une méthode de broyage (grinding). Une technique de cryobroyage a été mise au point par Chakraborty et al. (2005) mais n'a mené qu'à des diamètres de microfibrilles de l'ordre de 0,1 à $1 \mu \mathrm{m}$. D'autres méthodes ont également été répertoriées par Dufresne (2012) comme les ultrasons haute intensité ou l'electrospinning. Cependant, la technique la plus utilisée reste l'homogénéisation sous haute pression.

Les premiers travaux sur cette méthode à partir de pâte de résineux ont été développés par Herrick et al. (1983) et Turbak et al. (1983) en utilisant un homogénéisateur à lait. Il s'agit de forcer sous pression (55 MPa) le passage d'une suspension diluée de pâte (1-2\% en masse) à travers une mince fente. Les fibres sont alors soumises à d'importantes forces d'impact et de cisaillement qui provoquent un haut degré de fibrillation et conduisent à la production de MFC. Une autre technique d'homogénéisation est celle basée sur l'utilisation d'un microfluidiseur (Microfluidics Inc., USA), dans lequel la suspension de pâte est pompée à travers des chambres en forme de $\mathrm{Z}$ où elle est soumise également à des forces d'impact et de cisaillement. 
La méthode, efficace, présente cependant aussi des inconvénients : colmatages fréquents des chambres dans les appareils et consommation énergétique élevée due au nombre de cycles nécessaires (Herrick et al., 1983 ; Spence et al., 2011).

\subsection{Cellulose nanocristalline (CNC)}

La préparation de cellulose nanocristalline (cellulose nanocrystals, CNC) à partir de pâte de cellulose consiste en une hydrolyse acide contrôlée qui dissout préférentiellement les régions amorphes des fibres pour libérer les domaines cristallins (Dufresne, 2012). La CNC issue du bois se présente sous forme de bâtonnets de 3-5 nm de diamètre et d'une longueur de 100-200 nm (Brinchi et al., 2013). Ces bâtonnets sont composés à $100 \%$ de cellulose hautement cristalline (cristallinité entre $54 \%$ et $88 \%$ [Moon et al., 2011]). Il est possible d'obtenir de la CNC à partir de pâte chimique de type Kraft (blanchie ou non) en employant de l'acide sulfurique pour l'hydrolyse (Kontturi et al., 2009). C'est d'ailleurs souvent la technique employée pour obtenir des nanofibres directement à partir de pâte Kraft (Orts et al., 2005 ; Ioelovich et al., 2006 ; Stenstad et al., 2008). L'utilisation d'une hydrolyse enzymatique pour la production de CNC a été utilisée par Siqueira et al. (2010) en combinaison avec des traitements mécaniques. Selon les combinaisons de traitements appliquées, de la CNC était produite mais en combinaison avec de la MFC.

\section{LES PRÉTRAITEMENTS ENZYMATIQUES}

Dans le cas de production de MFC par homogénéisation à partir de fibres de cellulose, deux principaux inconvénients apparaissent. Le premier est celui posé par les colmatages fréquents de l'appareil qui,combinés avec le nombre de passages nécessaires (généralement de dix à vingt), impliquent de fréquentes interventions manuelles de débouchage. Ces colmatages imposent de travailler avec des concentrations initiales en cellulose très faibles (de 0,1 à $2 \%$ ). Le second est l'importante consommation énergétique engendrée par les multiples passages nécessaires de la suspension à travers les appareils. Cette consommation a été évaluée par Spence et al. (2011) selon l'appareil utilisé (broyeur, homogénéisateur ou microfluidiseur) et allait de $2280 \mathrm{kWh} \cdot \mathrm{t}^{-1}$ à $21900 \mathrm{kWh} \cdot \mathrm{t}^{-1}$, mais des valeurs supérieures à $30000 \mathrm{kWh} \cdot \mathrm{t}^{-1}$ ont aussi été observées (Nakagaito et al., 2004).

Afin de diminuer le nombre de passages nécessaires et donc aussi la consommation énergétique, des prétraitements chimiques ou enzymatiques peuvent être appliqués à la cellulose avant les traitements mécaniques. Les prétraitements chimiques étudiés comprennent l'hydrolyse acide, la carboxyméthylation ou encore une oxydation des fibres par du 2,2,6,6-tétraméthylpipéridine-1oxyl (TEMPO) (Mishra et al., 2012 ; Isogai, 2013). Ces méthodes font cependant appel à des réactifs couteux qui ne peuvent, dans certains cas, pas être recyclés. Bien que des prétraitements d'hydrolyse à l'acide puissent aussi être employés, cette technique est préférentiellement utilisée pour attaquer les zones amorphes de la structure dans un objectif de production de cellulose nanocristalline (Orts et al., 2005 ; Bondeson et al., 2006a ; Bondeson et al., 2006b ; Dufresne, 2006 ; Moran et al., 2008), dont les applications diffèrent de la MFC. L'utilisation d'enzymes lors de prétraitements (Henriksson et al., 2007 ; Pääkko et al., 2007 ; Zhu et al., 2011) présente quant à elle les avantages suivants : couts moins élevés des enzymes par rapport aux réactifs chimiques spécifiques (e.g. TEMPO), caractère écologique, absence d'installations spécifiques résistantes aux réactifs chimiques et possibilité plus aisée de récupération et valorisation des produits de l'hydrolyse. Ces avantages en font la méthode à favoriser dans le cadre d'un bioraffinage qui cherche à diminuer son impact sur l'environnement et à valoriser un maximum de ses coproduits.

L'hydrolyse enzymatique de la cellulose est un processus complexe du fait de la nature, structure et organisation des chaines de cellulose. Le mécanisme d'hydrolyse fait intervenir trois différents types d'enzymes, dont les actions indépendantes agissent en synergie pour former un système cellulase complet durant le processus d'hydrolyse (Rabinovich et al., 2002 ; Zhang et al., 2006 ; Liu et al., 2009). Ces trois groupes de cellulase se distinguent par leur activité :

- les endoglucanases (ou $\beta$-1,4-endoglucanases), qui hydrolysent au hasard les liaisons glucosidiques internes d'une chaine dans les zones amorphes, créant ainsi de nouvelles chaines de longueur variable de même que de nouvelles extrémités de chaines ;

- les exoglucanases qui hydrolysent les chaines pour libérer du glucose à partir d'une extrémité réductrice (glucanohydrolase) ou du cellobiose (dimère de glucose) à partir d'une extrémité non réductrice (cellobiohydrolase) ;

- les cellobiases (ou $\beta$-glucosidases) qui hydrolysent le cellobiose en glucose.

Il est donc possible de favoriser certaines activités en jouant sur la composition du mélange enzymatique utilisé. On distingue généralement les préparations de cellulases qui regroupent principalement les activités endoglucanase et cellobiohydrolase de celles à activité principalement cellobiase, bien que cellesci soient souvent utilisées en combinaison avec les 
premières. Les cellulases ont d'ailleurs déjà trouvé des applications dans le secteur papetier, principalement dans les étapes de raffinage des fibres ou de recyclage des papiers (Dufresne, 2012; Torres et al., 2012). La plupart des cellulases utilisées industriellement sont d'origine fongique ou microbienne et leur production est assurée par des entreprises spécialisées dans le domaine. Les principales souches fongiques utilisées sont Trichoderma spp. (T. reesei, T. viride et T. longibrachiatum) et Aspergillus spp. (A.niger et $A$.oryzae), mais il est également possible d'extraire des cellulases de cultures de Penicillium funiculosum, d'Humicola insolens, de Bacillus spp. ou de Saccharomyces spp. Il est important de noter que les complexes cellulasiques obtenus à partir de ces différentes souches possèdent généralement des propriétés variables. Ainsi, les cellulases produites par Aspergillus spp. sont principalement des endoglucanases avec de faibles quantités d'exocellulases, tandis que Trichoderma spp. produit surtout des cellobiohydrolases. Ces cellulases de différentes origines possèdent cependant un $\mathrm{pH}$ optimal assez similaire (aux environs de 5), alors que la température optimale est plus variable, allant de $45^{\circ} \mathrm{C}$ (souches d'Aspergillus sp.) à $65^{\circ} \mathrm{C}$ (souches de Penicillium sp.).

Bien que les enzymes soient déjà utilisées par les industries du secteur cellulosique, leur utilisation dans la production de nanocellulose (plus particulièrement de MFC) est en pleine croissance (Dufresne, 2012). Les sources de cellulose utilisées sont d'ailleurs variées, allant de la pâte à papier blanchie (Henriksson et al., 2007 ; Pääkkö et al., 2007 ; ) aux fibres de sisal (Agave sisalana Perrine) (Siqueira et al., 2010 ; Siqueira et al., 2011). Dans tous les cas, le traitement enzymatique est en réalité un prétraitement à l'application indispensable de forces mécaniques de cisaillement. Pääkkö et al. (2007) ont ainsi démontré qu'une étape de prétraitement enzymatique employant uniquement des endoglucanases sur de la pâte à papier blanchie facilitait l'étape d'homogénéisation en diminuant les cas de colmatages des chambres ainsi que la consommation énergétique des appareils mécaniques. La MFC produite possédait également des caractéristiques de tailles et de formes plus homogènes. Il faut cependant noter que la concentration et le type d'enzymes peuvent également affecter la longueur des fibres et la proportion de fibres dites fines. Une charge enzymatique peu élevée est toutefois suffisante pour faciliter la désintégration par homogénéisation, même si un meilleur résultat et une diminution des cas de colmatages sont obtenus en combinant ce prétraitement enzymatique avec un prétraitement mécanique visant à diminuer la longueur des fibres avant l'étape d'homogénéisation (Henriksson et al., 2007).

\section{POTENTIEL D'INTÉGRATION D'UNE LIGNE DE PRODUCTION DE NANOCELLULOSE DANS UNE USINE DE PÂTE KRAFT}

Comme la production de nanocellulose peut se faire en employant une partie de la pâte produite directement par une usine de pâte de type Kraft classique, l'intégration d'une telle ligne de production ne nécessite pas de modifier le fonctionnement des installations déjà existantes. Les principaux freins à son développement résident dans la variabilité des caractéristiques de la nanocellulose produite en fonction du processus employé (Klemm et al., 2011 ; Dufresne, 2012) et dans le contrôle des couts de production afin que ce produit puisse économiquement rivaliser avec certains dérivés de synthèse issus de la filière pétrochimique (agents de renfort, de densification, modificateurs rhéologiques, etc.). La mise au point de techniques de production assurant une uniformité des caractéristiques et restant économiquement compétitive est actuellement à l'étude (Nelson et al., 2014). Certaines firmes ont cependant déjà intégré, sur un site de production de pâte, une unité de valorisation de leur cellulose (en filament de cellulose [Kruger, Québec, Canada], en nanocellulose cristalline [Domtar, Québec, Canada]).

\section{MARCHÉS CIBLÉS ET POTENTIEL COMMERCIAL}

Le secteur papetier a bien compris l'avantage qu'il aurait à tirer de la production de nanocellulose et investit dans la recherche, comme en témoignent les usines pilotes construites en collaboration entre des industriels et des universités ou centres de recherche. Citons ainsi, de manière non exhaustive, certaines collaborations au Canada (Québec) (joint-venture entre Domtar Corporation et FPInnovations, Kruger et FPInnovations), aux États-Unis dans le Maine (University of Maine et USDA Forest Service) et le Wisconsin (US Forest Products Laboratory), en France (CTP et FCBA) ou encore en Suède (collaboration entre le Royal Institute of Technology et Innventia). Très récemment, SAPPI (South Africa Pulp and Paper Industries) et la Edinburgh Napier University faisaient part dans la presse de la création future d'une unité pilote après la découverte d'un nouveau procédé de fabrication de nanocellulose (Griffiths, 2015).

Plusieurs études de marché pour ces nanocelluloses ont été réalisées. Citons, parmi elles, celle de Future Markets (2015) ou celle de Shatkin et al. (2014) qui ont réalisé récemment une synthèse du marché potentiel de la nanocellulose et de ses applications selon les volumes utilisables (Tableau 3). Si la nanocellulose peut être utilisée dans des produits papetiers traditionnels 
Tableau 3. Applications potentielles de la nanocellulose selon les volumes utilisables (d'après Shatkin et al.,2014) - Potential applications of nanocellulose according usable volumes (adapted from Shatkin et al., 2014).

\begin{tabular}{lll}
\hline Applications à grands volumes & Applications à faibles volumes & Applications nouvelles ou émergentes \\
\hline Ciment & Panneaux muraux & Capteurs \\
Packaging & Isolation & Fibre de renforcement \\
Papier & Peinture & Filtration (air et eau) \\
Produits d'hygiène & Aérogels (industrie des gaz et huiles) & Modificateurs de viscosité \\
Textiles pour l'habillement & Aérospatial (structure et intérieur) & Purification \\
Automobile (carrosserie et intérieur) & & Cosmétiques \\
Remplacement plastique (packaging et & & Excipients \\
film) & & LED organique \\
& & Électronique flexible et recyclable \\
& & Photovoltaïque \\
& & Impression 3D \\
& & Films photoniques
\end{tabular}

(papiers, emballages), elle peut également l'être dans de nouveaux secteurs où la valeur ajoutée est importante pour une faible quantité de nanocellulose (e.g. pharmaceutique). Les applications nécessitant des volumes plus importants pourraient influencer la demande en nanocellulose et jouer sur les prix de celle-ci. Cowie et al. (2014) estiment ainsi raisonnable que la taille potentielle du marché mondial atteigne plus de 32 millions de tonnes de nanocellulose pour les marchés à grands volumes et plus de 2 millions pour les marchés à faibles volumes. À cela doit s'ajouter le marché des applications émergentes dont le volume est encore difficile à estimer.

\section{CONCLUSIONS}

L'utilisation d'enzymes dans l'industrie papetière est déjà d'actualité (désencrage, raffinage, etc.). Les entreprises spécialisées dans la production industrielle d'enzymes ont d'ailleurs déjà une gamme de mix à destination de cette industrie et ne cessent pas d'étoffer leur offre. Le concept d'IFBR est de plus en plus étudié et apparait comme une solution technologiquement et économiquement viable pour l'industrie papetière. Les techniques de production de nanocellulose (CNC et MFC) et les applications de celles-ci sont également déjà bien identifiées. Des applications nouvelles sont par ailleurs découvertes chaque année et le marché potentiel pour ces produits est estimé à plus de 34 millions de tonnes de nanocellulose. On remarque cependant que l'utilisation d'enzymes pour la production de nanocellulose reste confinée à des étapes de prétraitements, qui emploient principalement des endoglucanases. Or, l'objectif d'une IFBR est de valoriser au maximum ses produits et coproduits. Si la production de glucose destiné au secteur des biocarburants par hydrolyse enzymatique est déjà employée, d'autres produits d'hydrolyse peuvent également être valorisés. Par exemple, Vanderghem et al. (2012) ont montré la possibilité d'optimiser un processus d'hydrolyse de pâte à papier par des exoglucanases qui favorise la production de cellobiose. Avec l'intérêt croissant porté par le secteur papetier, la recherche future devrait s'orienter vers des méthodes de prétraitements enzymatiques qui permettent également la production et la valorisation de ce type de coproduits. Cette valeur ajoutée justifierait l'utilisation préférentielle des enzymes dans la production de nanocellulose et s'intégrerait dans la vision de valorisation maximale qui définit le concept d'IFBR.

\section{Bibliographie}

Bajpai P., 2013. Biorefinery in the pulp and paper industry. Burlington, MA, USA: Elsevier Science.

Brinchi L., Cotana F., Fortunati E. \& Kenny J.M., 2013. Production of nanocrystalline cellulose from lignocellulosic biomass: technology and applications. Carbohydr. Polym., 94, 154-169.

Bondeson D., Kvien I. \& Oksman K., 2006a. Strategies for preparation of cellulose whiskers from microcrystalline cellulose as reinforcement in nanocomposites. In: Oksman K. \& Sain M., eds. Cellulose nanocomposites: processing, characterization and properties. Washington: ACS Publications, 10-25.

Bondeson D., Mathew A. \& Oksman K., 2006b. Optimization of the isolation of nanocrystals from microcrystalline cellulose by acid hydrolysis. Cellulose, 13, 171-180.

Browne T. et al., 2013. Forest biorefinery: current state of the art. In: Stuart P.R. \& El-Halwagi M.M., eds. Integrated biorefineries: design, analysis and optimization. Boca Raton, FL, USA: CRC Press, 491-516.

Chaker A. et al., 2013. Key role of the hemicellulose content and the cell morphology on the nanofibrillation effectiveness of cellulose pulp. Cellulose, 20, 28632875 . 
Chakraborty A., Sain M. \& Kortschot M., 2005. Cellulose microfibrils: a novel method of preparation using high shear refining and cryocrushing. Holzforschung, 59, 102-107.

Christopher L.P., 2013. Integrated forest biorefineries: current state and development potential. In: Christopher L.P., ed. Integrated forest biorefineries: challenges and opportunities. Cambridge, UK: The Royal Society of Chemistry, 1-66.

Cowie J., Bilek E.M., Wegner T.H. \& Shatkin J.A., 2014. Market projections of cellulose nanomaterial-enabled products - Part 2: Volume estimates. TAPPI J., 13(6), 57-69.

Dufresne A., 2006. Comparing the mechanical properties of high performance polymer nanocomposites from biological sources. J. Nanosci. Nanotechnol., 6, 322330.

Dufresne A., 2012. Nanocellulose: from nature to high performance tailored materials. Berlin: Walter de Gruyter $\mathrm{GmbH}$.

Dufresne A., 2013. Nanocellulose: a new ageless bionanomaterial. Mater. Today, 16, 220-227.

FAO, 2013. Pulp and paper capacities, survey 2012-2017. Roma: FAO.

Fengel D. \& Wegener G., 1989. Wood. Berlin: Walter de Gruyter $\mathrm{GmbH}$.

Future Markets, 2015. The global market for nanocellulose, http://www.futuremarketsinc.com/the-global-marketfor-nanocellulose-to-2024/, (24/11/2015).

Griffiths S., 2015. New low-cost process to make nanocellulose, http://www.fstjournal.org/news/ new-low-cost-process-make-nanocellulose/670, $(24 / 11 / 2015)$.

Habibi Y., Lucia L.A. \& Rojas O.J., 2010. Cellulose nanocrystals: chemistry, self-assembly and applications. Chem. Rev., 110, 3479-3500.

Henriksson M., Henriksson G., Berglund L.A. \& Lindström T., 2007. An environmentally friendly method for enzyme-assisted preparation of microfibrillated cellulose (MFC) nanofibers. Eur. Polym. J., 43, 34343441 .

Herrick F.W., Casebier R.L., Hamilton J.K. \& Sandberg K.R., 1983. Microfibrillated cellulose: morphology and accessibility. J.Appl.Polym. Sci.: Appl. Polym. Symp., 37, 797-813.

Ioelovich M. \& Leykin A., 2006. Microcrystalline cellulose: nano-structure formation. Cellul. Chem. Technol., 40 , 313-317.

Isogai A., 2013. Wood nanocellulose: fundamentals and applications as new bio-based nanomaterials. J. Wood Sci., 59, 449-459.

Iwamoto S., Nakagaito A.N. \& Yano H., 2007. Nanofibrillation of pulp fibers for processing of transparent nanocomposites. Appl. Phys. A, 89, 461-466.

Jonoobi M. et al., 2015. Different preparation methods and properties of nanostructured cellulose from various natural resources and residues: a review. Cellulose, 22 , 935-969.

Jorfi M. \& Foster E.J., 2015. Recent advances in nanocellulose for biomedical applications. J. Appl. Polym. Sci., 132, 41719.

Klemm D. et al., 2011. Nanocelluloses: a new family of natur-based materials. Angew. Chem., 50, 5438-5466.

Kontturi E. \& Vuorinen T., 2009. Indirect evidence of supramolecular changes within cellulose microfibrils of chemical pulp fibers upon drying. Cellulose, 16, 65-74.

Lindström T. \& Aulin C., 2014. Market and technical challenges and opportunities in the area of innovative new materials and composites based on nanocellulosics. Scand. J. For. Res., 29, 345-351.

Liu H. et al., 2009. Visualization of enzymatic hydrolysis of cellulose using AFM phase imaging. Enzyme Microb. Technol., 45, 274-281.

Machani M., Nourelfath M. \& D'Amours S., 2014. A mathematically-based framework for evaluating the technical and economic potential of integrating bioenergy production within pulp and paper mills. Biomass Bioenergy, 63, 126-139.

Mishra S.P., Manent A.S., Chabot B. \& Daneault C., 2012. Production of nanocellulose from native cellulose various options utilizing ultrasound. Bioresources, 7, 422-436.

Moon R.J. et al., 2011. Cellulose nanomaterials review: structure, properties and nanocomposites. Chem. Soc. Rev., 40, 3941-3994.

Moran J.I., Alvarez V.A., Cyras V.P. \& Vazquez A., 2008. Extraction of cellulose and preparation of nanocellulose from sisal fibers. Cellulose, 15, 149-159.

Moshkelani M., Marinova M., Perrier M. \& Paris J., 2013. The forest biorefinery and its implementation in the pulp and paper industry: energy overview. Appl. Therm. Eng., 50, 1427-1436.

Nakagaito A.N. \& Yano H., 2004. The effect of morphological changes from pulp fiber towards nanoscale fibrillated cellulose on the mechanical properties of high-strength plant fiber based composites. Appl. Phys. A, 78, 547-552.

Nelson K. \& Retsina T., 2014. Innovative nanocellulose process breaks the cost barrier. TAPPI J., 13(5), 19-23.

Orts W.J. et al., 2005. Application of cellulose microfibrils in polymer nanocomposites. J. Polym. Environ., 13(4), 301-306.

Pääkkö M. et al., 2007. Enzymatic hydrolysis combined with mechanical shearing and high-pressure homogenization for nanoscale cellulose fibrils and strong gel. Biomacromolecules, 8, 1934-1941.

Postek M.T. et al., 2011. Development of the metrology and imaging of cellulose nanocrystals. Meas. Sci. Technol., 22, 024005.

Rabinovich M.L., Melnik M.S. \& Bolbova A.V., 2002. The structure and mechanism of action of cellulolytic enzymes. Biochemistry Mosc., 67, 850-871. 
Rafione T., Marinova M., Montastruc L. \& Paris J., 2014 The green integrated forest biorefinery: an innovative concept for the pulp and paper mills. Appl. Therm. Eng., 73, 74-81.

Shatkin J.A., Wegner T.H., Bilek E.M. \& Cowie J., 2014. Market projections of cellulose nanomaterial-enabled products - Part 1: applications. TAPPI J., 13(5), 9-16.

Siqueira G. et al., 2010. Morphological investigation of nanoparticles obtained from enzymatic and acid hydrolysis of sisal fibers. Cellulose, 17, 1147-1158.

Siqueira G. et al., 2011. Mechanical properties of natural rubber nanocomposites reinforced with cellulosic nanoparticles obtained from enzymatic and acid hydrolysis of sisal fibers. Cellulose, 18, 57-65.

Siró I. \& Plackett D., 2010. Microfibrillated cellulose and new nanocomposite materials: a review. Cellulose, 17, 459-494.

Spence K. et al., 2011. A comparative study of energy consumption and physical properties of microfibrillated cellulose produced by different processing methods. Cellulose, 18, 1097-1111.

Stenstad P., Andresen M., Tanem B.S. \& Stenius P., 2008. Chemical surface modification of microfibrillated cellulose. Cellulose, 15, 35-45.

Stevanovic T. \& Perrin D., 2009. Chimie du bois. Lausanne, Suisse: Presses polytechniques et universitaires romandes.

Taniguchi T. \& Okamura K., 1998. New films produced from microfibrillated natural fibres. Polym. Int., 47, 291294.
Torres C.E., Negro C., Fuente E. \& Blanco A., 2012. Enzymatic approaches in paper industry for pulp refining and biofilm control. Appl. Microbiol. Biotechnol., 96, 327-344.

Turbak A.F., $\quad$ Snyder F.W. \& Sandberg K.R., 1983. Microfibrillated cellulose: a new cellulose product. Properties, uses and commercial potential. J. Appl. Polym. Sci.: Appl. Polym. Symp., 37, 815-827.

Vanderghem C. et al., 2012. Effect of physiochemical characteristics of cellulosic substrates on enzymatic hydrolysis by means of a multi-stage process for cellobiose production. Appl. Biochem. Biotechnol., 166, 1423-1432.

Zhang X., Paice M.G. \& Deng J., 2011. Modify existing pulp and paper mills for biorefinery operations. In: Zhu J., Zhang X. \& Pan X., eds. Sustainable production of fuels, chemicals and fibers from forest biomass. Washington: ACS Publications, 395-408.

Zhang Y.H.P., Himmel M.E. \& Mielenz J.R., 2006. Outlook for cellulose improvement: screening and selection strategies. Biotechnol. Adv., 24, 452-481.

Zhu J.Y., Sabo R. \& Luo X., 2011. Integrated production of nano-fibrillated cellulose and cellulosic biofuel (ethanol) by enzymatic fractionation of wood fibers. Green Chem., 13, 1339-1344.

(54 réf.) 